\title{
A interação entre as pessoas com síndrome de Down e seus irmãos: um estudo exploratório
}

\author{
The interaction between people with Down syndrome \\ and their siblings: an exploratory study
}

Bruna Rafaela de Batista ${ }^{1}$

Márcia Duarte ${ }^{2}$

Fabiana $\mathrm{Cia}^{2}$
${ }^{1}$ Licenciatura em Educação Especial, Universidade Federal de São Carlos (UFSCar). Rodovia Washington Luis Km 235, Jardim Guanabara. 13565 905 São Carlos SP Brasil. bru_tifa@yahoo.com.br ${ }^{2}$ Departamento de

Psicologia, UFSCar. São Carlos SP Brasil.

\begin{abstract}
The presence of a disabled person causes transformations in the family that demand a redefinition of the role of each member. Siblings, like all other members, experience frustration, acceptance, guilt and adaptation. In this respect, an attempt was made to; (a) analyze the interaction between a sibling with standard development and a sibling with Down syndrome; (b) identify what information and reaction the siblings with standard development have regarding the diagnosis of Down syndrome; (c) verify whether or not there were changes in the family context and also changes in their own lives after the birth of the sibling with Down syndrome. The survey was conducted through interviews, the participants being seven children with standard development having siblings with Down syndrome. The study showed that the siblings, as well as the other family members, experience processes of acceptance, confusion and other complex emotions related to the disability, albeit they are not as marked as those experienced by their parents. A fact revealed in this study is the importance of support groups for siblings, seeking to handle issues such as prejudice and adverse feelings towards disability. The siblings with standard development must have correct information, as well as support, in order to foster healthy interaction between siblings.
\end{abstract}

Key words Siblings, Down syndrome, Family
Resumo A presença de uma pessoa com deficiência provoca transformações na família que exigem, de cada membro, redefinição de papéis. $O$ irmão, como todos os outros membros, passa por processos de frustração, aceitação, culpa e mudanças. Nesse sentido, objetivou-se (a) analisar a interação entre o irmão com desenvolvimento típico e o com sindrome de Down; (b) identificar que informação e reação os irmãos com desenvolvimento típico possuem sobre o diagnóstico da sindrome de Down; (c) verificar se houve ou não modificações no contexto familiar e em suas próprias vidas após o nascimento do irmão com síndrome de Down. A pesquisa, realizada por meio de entrevistas, teve como participantes sete sujeitos com desenvolvimento típico, irmãos de pessoas com sindrome de Down. O estudo demonstrou que os irmãos, assim como os demais agentes familiares, passam por processos de aceitação, confusão e por várias outras complexidades com a qual a deficiência está atrelada, mas que estas não são tão marcantes quanto aos de seus pais. Um indicativo apontado no estudo se refere à importância de grupos de apoio para irmãos, que visem trabalhar questões como preconceito e sentimentos aversivos perante a deficiência. Aqueles com desenvolvimento típico precisam de informações corretas, bem como apoio, visando favorecer a interação entre irmãos. Palavras-chave Irmãos, Síndrome de Down, Família 


\section{Introdução}

A família constitui uma instituição socialmente construída que se modificou ao longo dos anos com dinâmicas de relação variadas, cujo funcionamento muda em decorrência de qualquer alteração que venha a ocorrer em um de seus membros ${ }^{1}$.

O nascimento de uma criança com síndrome de Down em uma família pode gerar situações complexas e resultantes da falta de preparo e informação adequada para lidar com os sentimentos que possivelmente surgirão nesse momento. A síndrome de Down é uma desordem cromossômica que se caracteriza pela existência de um cromossomo extra ou parte de um, causando uma triplicação ao invés da duplicação do material genético referente ao par $21^{2}$.

Embora cada família seja única, uma experiência como o nascimento de uma criança com síndrome de Down traz a necessidade de adaptação e reações que se assemelham entre algumas famílias. As expectativas da família necessitam ser revistas, pois se trata de uma situação inesperada, exigindo o ajuste às mudanças de planos ${ }^{3}$.

Para Petean e Suguihura ${ }^{4}$, a família passa por um processo de superação à aceitação do filho com deficiência, até conseguir instituir um ambiente familiar propício para a criança. Mesmo que se possa identificar a vivência de diversos sentimentos pelos pais e mães, a reação de choque, a culpa, a raiva e a tristeza são comuns a todos. Frente aos irmãos, a notícia da deficiência parece ter um efeito menos dirigido para o seu desenvolvimento socioemocional, sendo poucas as manifestações de sentimentos extremos, ficando apenas no nível da tristeza, quando são expressos.

$\mathrm{Chacon}^{5}$, em seu estudo, apontou que as diferenças no momento em que cada pai sabe da deficiência podem estar relacionadas à disposição que ele tem em aceitar aquilo que está vivenciando com relação às necessidades do filho. $O$ autor afirmou ainda, que a maioria das famílias se sentiu chocada ao receber a notícia sobre a deficiência do filho.

Estudos produzidos sobre famílias de pessoas com deficiência apontam que quanto mais informação a família tiver sobre a deficiência, mais preparada estará para a busca de suportes e apoios que contribuam para o desenvolvimento de seu familiar ${ }^{1-6}$.

No entanto, segundo Mendes et al. ${ }^{7}$, os profissionais da saúde geralmente não estão preparados para informar e orientar a família sobre as condições da criança com deficiência.
Porém, com informações necessárias, o ambiente familiar estará mais preparado para lidar com as situações geradas com a chegada de um de seus integrantes com deficiência, a qual todos os membros poderão participar ativamente em seu desenvolvimento, visto que o mundo familiar da criança vai muito além da interação mãe-criança. A interação com outros membros familiares, como pais, irmãos e avós desempenha um papel fundamental no desenvolvimento das crianças ${ }^{8}$.

Mediante a importância dos integrantes familiares para com a pessoa com deficiência, notase que a influência familiar no desenvolvimento, comportamento e formação da personalidade do deficiente se dá, primordialmente, por meio das relações estabelecidas. As interações e as relações entre os membros do sistema familiar mostram que o desenvolvimento da pessoa não pode ser isolado do da família ${ }^{1}$.

Considerando toda complexidade que cerca a questão da interação familiar com a criança com síndrome de Down, seria interessante situar a princípio estudos sobre esta temática e se a questão da interação entre irmãos já vem sendo objeto das pesquisas.

Silva e Dessen ${ }^{1}$ apontam que as relações mais estudadas referentes no contexto familiar são entre mãe-criança (39\%), quando comparadas às outras interações como pai-criança e criança -irmão. Os estudos sobre irmãos em sua maioria são realizados com díades de irmãos, sendo que, em muitas famílias, há mais de dois filhos?. Entre os estudos na literatura que se referem à interação de irmãos de pessoas com deficiência, pode-se destacar Powell e Ogle ${ }^{10}$; Silva ${ }^{11}$; König ${ }^{12}$; Van Riper ${ }^{13}$; Petean e Suguihura ${ }^{4}$; Nunes e Aielo ${ }^{14}$; Messa e Fiamenghi ${ }^{15}$.

Em um estudo realizado por Van Riper ${ }^{13}$, cujo objetivo foi analisar as relações entre as exigências, os recursos, o enfrentamento, a comunicação de resolução de problemas de família, e o bem-estar dos irmãos em 41 famílias de crianças com síndrome de Down, os resultados indicaram que os irmãos tinham autoconceitos favoráveis, e perante os relatos das mães, estes irmãos eram socialmente competentes, com uma baixa incidência de problemas de comportamento.

Neste mesmo sentido, Nunes e Aiello ${ }^{14} \mathrm{ca}-$ racterizaram a interação entre díades de irmãos, o estudo comparou os desempenhos em jogos competitivos e cooperativos, e comparou os níveis de estresse e as estratégias de enfrentamento em situações cotidianas. Os resultados mostraram que o relacionamento entre os irmãos, no qual há um deficiente, é menos íntimo, diferente 
em padrões de cuidado, caracterizado por assimetrias de papéis, não havendo diferenças significativas entre as díades quanto à frequência de estressores. Os resultados também indicaram que os irmãos com desenvolvimento típico não apresentam ações negativas em direção àquele com deficiência intelectual.

A forma que um irmão com desenvolvimento típico responde à presença do outro com deficiência na família dependerá de vários fatores, alguns estudos ${ }^{16-18}$ apontam: idade dos irmãos e a disponibilidade de apoio social da família.

Perante o exposto, pode-se perceber que estudos que investigam a interação entre irmãos são importantes, especialmente, estudos que envolvem interações entre irmãos de pessoas com síndrome de Down.

Diante deste contexto o estudo partiu das seguintes questões de pesquisa: Quais são as interações diárias e sociais entre os irmãos com síndrome de Down e com desenvolvimento típico? Quais as informações que o irmão com desenvolvimento típico tem sobre o seu irmão com síndrome de Down? Quais as modificações na dinâmica familiar e na própria vida após o nascimento do irmão com síndrome de Down?

Nesse sentido, os objetivos do presente estudo foram: (a) analisar a interação entre o irmão com desenvolvimento típico e o com síndrome de Down; (b) identificar que informação e reação os irmãos com desenvolvimento típico possuem sobre o diagnóstico da síndrome de Down; (c) verificar se houve ou não modificações no contexto familiar e em suas próprias vidas após o nascimento do irmão com síndrome de Down.

\section{Método}

O presente estudo faz parte de um Projeto de Pesquisa aprovado pelo Comitê de Ética da Universidade Federal de São Carlos. Perante o ponto de vista metodológico teve-se como foco a pesquisa de cunho qualitativo, que segundo Ludke e André ${ }^{19}$ permite a análise e a descrição dos dados de forma articulada, tendo o ambiente como fonte direta e o pesquisador como seu principal instrumento. Trata-se de um estudo exploratório, em que foi utilizado um roteiro de entrevista semiestruturado. Como a pesquisa foi desenvolvida com poucos participantes não se pode generalizar seus resultados, estudos com amostras ampliadas e com procedimentos diversos de coleta de dados são aconselháveis.

\section{Composição do grupo}

Os participantes foram sete irmãos, que atendiam o critério: ser irmão com desenvolvimento típico de pessoa com síndrome de Down, e ter idade mínima de 17 anos. Os critérios de exclusão se basearam na idade estabelecida, tendo em vista a compreensão do roteiro de questões aplicado.

A partir das informações advindas de questões para identificação, sabe-se que, dos sete participantes, cinco eram do sexo feminino e dois do masculino e tinham idade mínima de 17 anos e máxima de 35 anos. Três dos participantes moravam sozinhos e quatro com a família, a maioria destes (6) já possuía um vínculo empregatício, com as profissões de piloto, servidor público, professor, recepcionista, coordenador de processamento de produtos e atendente de supermercado. Neste estudo, não foi realizado um aprofundamento em relação aos dados socioeconômico dos participantes.

Mesmo não sendo critério de participação, todos os participantes eram mais velhos do que seus irmãos com síndrome de Down, com a diferença de idade variando entre sete e 17 anos. Cabe ressaltar também, que quatro dos participantes eram provenientes de famílias que frequentavam grupos de apoio para familiares de pessoas com deficiência.

\section{Procedimentos para coleta dos dados}

A coleta de dados foi realizada por meio de encontros que ocorreram em uma sala do prédio da Secretaria Municipal de Educação de uma cidade do interior do estado de São Paulo. Nesses encontros, foi realizada uma entrevista com cada participante individualmente, a qual foi assegurado um local sem interferências, visando garantir a privacidade e o anonimato dos depoimentos.

As entrevistas tiveram em média a duração de 30 minutos, sendo gravadas em áudio e transcritas na íntegra, para posterior análise. De acordo com Manzini ${ }^{20}$, a entrevista como método de coleta de dados pode fazer emergir informações de forma mais livre e não condicionadas a uma padronização.

\section{Instrumento}

O instrumento utilizado para a coleta de dados foi um roteiro de entrevista semiestruturado, que, segundo Manzini ${ }^{20}$, permite abordar o tema que se pretende investigar por meio de perguntas 
principais e complementadas por outras inerentes ao momento da entrevista.

A elaboração do roteiro foi realizada pela pesquisadora e por sua orientadora, sendo este avaliado pelo Grupo de Estudos e Pesquisa sobre a Escolarização da Pessoa com Deficiência - GEPEPD. Aproximadamente 5 pessoas avaliaram o roteiro, contribuindo com sugestões para a adequação do mesmo.

O roteiro, assim, se constituiu por 24 questões, dividido em Caracterização dos irmãos respondentes e em quatro eixos temáticos, a saber: (a) reação e informações sobre o diagnóstico; (b) modificações no contexto familiar; (c) rotina diária; e (d) interação entre os irmãos.

\section{Procedimentos de análise dos dados}

A análise das entrevistas foi de cunho qualitativo, por meio de trechos das falas orais. Os dados foram analisados mediante a leitura minuciosa das entrevistas e organizadas em temas que se aproximavam do objetivo da pesquisa. Segundo Vilelas ${ }^{21}$, a análise por temáticas consiste em operações de desmembramento do texto em unidades, mediante reagrupamentos analógicos, visando descobrir os núcleos de sentidos que compõem uma comunicação.

\section{Resultados e discussão}

A partir da análise dos dados foram identificados quatro eixos temáticos, sendo eles:

\section{1) Reação e informações sobre o diagnóstico da síndrome de Down}

No primeiro bloco foram relatadas as reações e as informações que os irmãos com desenvolvimento típico atribuem ao diagnóstico da síndrome de Down. Os sete participantes relataram que receberam a notícia sobre a síndrome de Down logo após o nascimento do irmão, sendo transmitida pelos próprios pais. Quando os participantes receberam a notícia, suas idades variavam entre sete e 17 anos. Independente da fase (infância, adolescência e juventude) em que se encontravam ao receberem a notícia, os participantes relataram passar pelas mesmas reações, como, de no início não compreender sobre o que era síndrome de Down e a de vivenciar sentimentos aversivos como os de tristeza, susto, ou normalidade.

Frente a esses dados, pode-se questionar sobre a não compreensão dos participantes em relação à síndrome de Down e se o momento correto da notícia aos irmãos deveria ser logo após o nascimento. Seria oportuno esse momento para a notícia? Indicativos estes que demonstram a necessidade de pesquisas que contribuam nesse sentido.

No estudo de Petean e Suguihura ${ }^{4}$, em que a idade dos participantes ao nascimento do irmão com síndrome de Down variou entre três a 15 anos, os resultados frente à notícia da deficiência demonstraram que para os irmãos com desenvolvimento típico esta parece ter um efeito menos dirigido, seis dos participantes disseram terem reagido normalmente ao receberem a notícia, dois relataram de não se lembrarem da reação, e três, que foi ruim, pois rejeitaram o irmão. $\mathrm{O}$ que difere deste estudo, ao que se refere à falta de recordação ou rejeição ao irmão com síndrome de Down.

Ainda sobre essa temática, outro aspecto que se pode verificar nos relatos dos participantes, foi a necessidade de clareza sobre a pronuncia do diagnóstico da síndrome pelos profissionais de saúde aos pais. Em um dos relatos, o participante descreveu que sua mãe lhe deu a notícia, dizendo que o médico apresenta o filho com síndrome de Down como uma pessoa totalmente diferente (pé, mão e orelhas). Esse relato da transmissão demonstra a importância do cuidado do profissional de saúde ao relatar a notícia do diagnóstico aos pais, que talvez, devido à emoção do momento, transmita a notícia para seus filhos de forma menos adequada, corroborando com os estudos de Petean e Suguihura ${ }^{4}$, Buscaglia ${ }^{22}$ e Chacon ${ }^{5}$.

De fato, segundo Buscaglia ${ }^{22}$, normalmente, o nascimento de um membro da família com deficiência é constituído como um acontecimento novo e inesperado, e seria imprescindível que a família recebesse um diagnóstico médico compreensível, que suavizasse os sentimentos de culpa, medo e incerteza, que acontecem com a chegada da criança.

Para Petean e Suguihura4: "É importante que eles tenham informações claras e seguras sobre o que está ocorrendo com o seu irmão, para que não fantasiem criando expectativas ou preconceitos que muito dificultarão o relacionamento entre eles, podendo também comprometer o estabelecimento do vínculo fraterno".

Como apontado pelas autoras, seria necessário que os irmãos recebessem informações claras, que possam minimizar o impacto da notícia. Pois, sabe-se que sempre haverá um impacto ao se receber uma notícia de que se tem um irmão com deficiência, uma vez que esta condição, em nossa sociedade, é permeada de estereótipos. 

ção de cuidado irmão-irmão. Os demais participantes (2) relataram existir essa obrigação de relação de cuidado devido ao compromisso de trabalho dos pais. Este fato difere do apontado pela literatura; por exemplo, no estudo de Petean e Suguihura ${ }^{4}, 90,9 \%$ dos participantes relataram ter responsabilidade pelas atividades de vida diária com o irmão com síndrome de Down.

Os irmãos com desenvolvimento típico também foram questionados se acreditavam ter maior responsabilidade do que amigos que não possuem irmãos com deficiência. Dois participantes relataram não possuir maiores responsabilidades, pois acreditavam que o relacionamento de irmãos, tanto com desenvolvimento típico, quanto atípico é permeado por atividades de cuidado. Os demais participantes (5) referiram à síndrome como fator que propicia esse aumento de responsabilidade. Tal fato, vai ao encontro do que a literatura evidencia, que aqueles que têm irmãos com deficiência, geralmente, tornam-se mais maduros e assumem mais responsabilidades do que os que não os têm ${ }^{3,24}$.

Outra questão abordada aos participantes foi se o relacionamento que seus pais têm com o irmão com síndrome de Down era diferente da relação com eles. Três participantes afirmam que há diferença, devido às necessidades que a síndrome impõe, como, por exemplo, mais cuidados em relação ao tratamento de saúde, ou a severidade e a proteção de seus pais. Quatro participantes acreditavam que a relação se dá sem diferenças significativas. Todos os participantes relataram que mesmo com diferenças ou não no relacionamento com seus pais, a família sempre busca estabelecer diálogo sobre o desenvolvimento do irmão com síndrome de Down, promovendo alternativas para os problemas que surgem nas escolas e nos atendimentos clínicos. Para Núñes ${ }^{25}$, a presença de uma pessoa com deficiência pode fortalecer os vínculos familiares e tornar seus integrantes mais maduros. O que pode-se notar nos relatos deste estudo, a qual a família busca estabelecer cooperação, fortalecendo seus vínculos em prol do desenvolvimento do irmão com síndrome de Down.

Também em relação ao tipo de relacionamento entre os pais e os participantes, estes foram questionados se gostariam que a relação com os pais e o irmão com síndrome de Down fosse diferente, tendo como respostas de três participantes que sim, no que se refere a mimar ou ser duro demais com o que tem síndrome de Down. Quatro participantes disseram que não, pois acreditavam que a relação de pais-filhos é estabelecida dessa maneira.

Assim, como vem sendo apontado na literatura, a função fraterna de irmãos é uma das estruturais do contexto familiar, de ajuda recíproca, de defesa dos direitos, colaboração e assistência em nível de igualdade ${ }^{26}$.

A compreensão sobre a situação familiar, ou mesmo sobre a responsabilidade com o irmão com síndrome de Down, acontece também pela maturidade dos com desenvolvimento típico que se encontravam entre o final da adolescência e na fase adulta. Cabe ressaltar, que se este estudo fosse realizado em outra faixa etária, os dados poderiam ser diferentes, visto que há uma tendência de se aceitar melhor as situações conforme o desenvolvimento maturacional.

Ainda nesse eixo, os participantes foram solicitados a falarem sobre os pontos positivos e os negativos de ter um irmão com síndrome de Down. Em relação aos positivos, os temas abordados foram: carinho, sinceridade, lidar com a diferença, companheirismo, aprendizado, mudanças, conhecimento e responsabilidade. Esses dados corroboram o estudo de Mayer ${ }^{27}$, realizado com mães de crianças com síndrome de Down, que ao relatarem sobre seus filhos também utilizaram adjetivos positivos, assim como os participantes deste estudo.

No que se refere a pontos negativos, somente um participante diz não existir. Nas falas dos demais participantes, pode-se verificar aspectos como: excesso de atendimento, preconceito, preocupação com a saúde, educação, desenvolvimento físico e cognitivo e falta de profissionais qualificados.

Mediante os dados apresentados pode-se observar que os participantes descrevem diversos pontos positivos em ter um irmão com síndrome de Down e que os negativos são diretamente proporcionados pelas dificuldades e pelo cuidado que a síndrome impõe, assim como o olhar da sociedade perante essas pessoas. Os fatores positivos e negativos fazem com que os irmãos com desenvolvimento típico tendam a ser tolerantes, empáticos, e criem maior senso de laços familiares ${ }^{24-28}$.

De acordo com os relatos, um dos temas abordados no ponto negativo em se ter um irmão com síndrome de Down foi o preconceito, aspec- 
to questionado a eles também. Quatro participantes relataram ter sofrido preconceito devido à síndrome do irmão, e três não. Tais resultados podem ser indicativos que os irmãos de pessoas com deficiência, necessitam também de que favoreçam a interação dos mesmos, assim como que se trabalhe com as questões que envolvem a deficiência e seus sentimentos e percepção perante a mesma.

Segundo os relatos dos participantes pode-se perceber que a deficiência ainda não é um fator totalmente aceito pela sociedade, na qual algumas pessoas demonstram não ter conhecimento sobre e julgam desnecessariamente, provocando em alguns desses irmãos sentimentos de tristeza, revolta, normalidade, entre outros.

Para Messa e Fiamenghi ${ }^{15}$, a adaptação dos irmãos a situações de deficiência dependerá de aspectos, como: recursos pessoais, história de vida, significado que a deficiência é compartilhada no sistema familiar, histórico das relações familiares, como também, disponibilidade de recursos de apoio. Alguns projetos que são desenvolvidos com famílias de pessoas com síndrome de Down têm se mostrado promissores para melhorar a qualidade de vida das famílias, oferecer apoio informacional e emocional, como também apoiar diretamente na autonomia e independência dos indivíduos com essa condição $0^{29,30}$.

Finalizando esse eixo, os participantes foram questionados sobre se sentirem responsáveis pelo irmão com síndrome, no caso de falecimento dos pais. Todos participantes responderam que sim, sendo já ser plano futuro de alguns. Os dados apresentados indicam que existe grande responsabilidade fraterna entre os irmãos, o que corrobora com o estudo de Silva ${ }^{11}$, que indica preocupação dos irmãos com desenvolvimento típico, quanto à responsabilidade de assumir o deficiente na ausência dos pais.

Novamente, esses dados são indicativos de que a idade dos participantes do estudo pode ter influenciado em suas tomadas de decisões, podendo ser um fator que favorece assumir a responsabilidade do irmão com síndrome de Down e a de cooperação familiar.

\section{3) Rotina diária}

Quanto aos aspectos sobre rotina diária, três participantes que moravam sozinhos informaram que a convivência com o irmão com síndrome de Down acontece somente no período noturno ou aos finais de semana, os participantes justificaram residir em outra cidade e passar grande tempo fora de casa devido a compromissos pessoais. Os demais participantes (4), mesmo morando juntos com o irmão com síndrome de Down, também apontaram fatores que interferem na rotina diária, como os constantes compromissos que o irmão possui, como atendimentos e atividades extras ou os seus próprios compromissos: trabalho e estudo.

De modo geral, a maioria dos participantes relatou participar de alguma forma da rotina de compromissos de seus irmãos e no convívio social com a família. Para Cuskelly ${ }^{31}$ e Lobato ${ }^{32}$, ter um irmão com deficiência pode provocar no desenvolvimento do irmão típico aspectos como: responsabilidade, confiança, paciência, independência e ligação afetiva com a família, fatores estes que foram observados nos relatos dos participantes deste estudo.

Em relação à opinião dos participantes se a síndrome de Down influencia na rotina diária da família, cinco participantes apontaram que não, e dois consideraram que a modificou em relação a passeios e aos finais de semana, pois precisavam permanecer em casa para cuidados com o irmão, porém, esses participantes atualmente moram sozinhos, e descreveram que suas rotinas não têm sido tão influenciadas como quando moravam com seus pais.

Os relatos destes participantes deixam transparecer que quando existe alteração da rotina diária, essa se dá pela preocupação ou responsabilidade de cuidar do irmão com síndrome na ausência de seus pais. Para a maioria dos participantes (5), ter um irmão com síndrome não influencia diretamente suas rotinas, o que difere do estudo de Petean e Suguihura ${ }^{4}$, a qual a maioria dos participantes $(82 \%)$ relatou que a presença de um irmão com essa condição acarretou mudanças significativas em suas vidas, como acréscimo de responsabilidade, discussões, comportamento/humor e dinâmica da família.

\section{4) Interação entre os irmãos}

Aqueles com desenvolvimento típico relataram qualidades, tais como o irmão com síndrome de Down ser: carinhoso, comunicativo, alegre e companheiro. Esses dados corroboram o estudo de $\mathrm{Mayer}^{27} \mathrm{com}$ as falas de mães ao descreverem seus filhos com síndrome de Down como carinhosos e alegres. Foi apontado também por quatro participantes comportamentos de hiperatividade e birra.

Pelos relatos pode-se notar que os irmãos possuem um grande laço afetivo, e mesmo com 
alguns comportamentos inadequados daqueles com síndrome de Down, o afeto e o vínculo não são comprometidos. Segundo a literatura, a relação fraterna entre irmãos é uma das relações mais perdurável na vida das pessoas, que passa por fases ao longo da vida, e que se desenvolve na medida em que eles crescem ${ }^{4}$.

Quanto à interação com o irmão com síndrome de Down, os participantes foram unânimes em relatar que é tida como boa. Observou-se nos relatos dos participantes que não residem juntos (3) que estes fazem o possível para estar presente como, por exemplo, ligações telefônicas ou fazendo atividades nos finais de semana. Os demais participantes (4) relatam que mesmo tendo maior convivência com o irmão com síndrome de Down, os momentos de interação acontecem em horários vagos (serviço e escola), sendo também, o maior tempo de interação nos finais de semana. Como já apontado no eixo rotina diária.

Esses resultados demonstram que, segundo a maioria dos participantes, a interação com o irmão com síndrome de Down é muito boa e os participantes procuram sempre estar presentes na vida dos mesmos. Quando estão juntos, estabelecem uma interação com afetividade, companheirismo, proteção e cuidado com o irmão. Corroborando o estudo de Volling e Blandon ${ }^{33}$, que descreve que a relação fraterna entre irmãos pode ser marcada por conflitos, mas ser afetiva e íntima.

Os participantes também foram questionados sobre sentimentos de vergonha e aversão. Em relação a sentir vergonha do irmão com síndrome de Down, apenas três participantes disseram que sim, na juventude ou devido a comportamentos inadequados, como correr e gritar, já em relação a sentimentos de aversão todos disseram inexistir.

Mediante os relatos, pode-se notar que os sentimentos negativos como vergonha e aversão não estão tão presentes na vida dos participantes, e quando há existência é devido aos maus comportamentos ou ao olhar da sociedade perante a deficiência.

No estudo de Naylor e Prescott ${ }^{34}$ foi apontada a importância de grupos de apoio para irmãos de crianças deficientes, que tem contribuído para amenizar tais sentimentos, ajudando no aumento da autoestima do irmão, propiciando experiências de sentimentos positivos juntamente com outras pessoas que possuem irmãos com deficiência. Novamente os dados apresentam a relevância de grupos e projetos que colaborem para o empoderamento das famílias de pessoas com síndrome de Down, assim como já apresentado também no item Modificações no contexto familiar.

Por fim, o último questionamento aos participantes foi em relação à escolha de ter ou não um irmão com síndrome de Down, a qual os relatos foram positivos em o ter. Observou-se nos relatos dos participantes que ter um irmão com síndrome de Down significa ter um presente divino e ter uma pessoa especial em suas vidas, os irmãos assim como outros familiares tendem responder a ocorrência da deficiência de um ponto de vista religioso, visando compreender e aceitar tal fato. Para Brunhara e Petean ${ }^{35}$, ao discutirem tal aspecto, analisam a referência ao ato divino como resignação, sendo esta uma renúncia espontânea de uma graça.

Com os resultados e as discussões apresentadas neste estudo, observou-se que o fator da maturidade dos participantes pode ter influenciado os resultados, e que se este estudo fosse realizado em outra faixa etária, outros dados poderiam ser apresentados. Além disso, parte deste estudo é retrospectivo, assim, o que os irmãos com desenvolvimento típico pensam e analisam hoje é diferente e influenciador do que viveram.

Cabe ressaltar que alguns desses participantes eram provenientes de famílias que participavam de grupos de apoio, o que contribuí para a percepção quanto ao irmão com síndrome de Down e a relação familiar constituída. Também o fato da maioria dos participantes ser do sexo feminino pode ter favorecido as respostas quanto ao cuidar.

\section{Considerações finais}

A proposta do estudo foi apresentar a caracterização da interação entre irmãos com desenvolvimento típico e com síndrome de Down, e refletir sobre as modificações na dinâmica familiar e na vida dos primeiros, com a chegada do segundo.

O estudo demonstrou que os irmãos, assim como os demais agentes familiares, passam por processos de aceitação, confusão e por várias outras complexidades com a qual a deficiência está atrelada, mas que estas não são tão marcantes quanto para seus pais.

Um indicativo apontado no estudo se refere à importância de grupos de apoio para irmãos, que visem trabalhar questões como preconceito e sentimentos aversivos perante a deficiência. Os sujeitos com desenvolvimento típico precisam de informações corretas, bem como apoio, visando favorecer a interação entre irmãos. 
Os resultados demonstraram que a interação com o irmão com síndrome de Down é boa e os com desenvolvimento típico procuram sempre estar presente na vida dos mesmos, porém há vários aspectos que contribuíram ou não para essa interação, como já apontado neste estudo e na literatura.

Sabe-se que o estudo não se encerra, pois há a necessidade de mais pesquisas na busca de novas inquietações e de respostas em relação às interações fraternas de pessoas com deficiência. O estudo poderá ser oferecido em outras populações, de diferente faixa etária, no intuito de analisar a influência de tais fatores sobre as variáveis estudadas.

\section{Colaboradores}

BR Batista participou da elaboração e organização geral do artigo, realização da análise de conteúdo, apresentação, discussões e interpretações. M Duarte participou da definição e orientação da metodologia do trabalho e revisão geral do artigo. F Cia fez o aprofundamento da revisão teórica e participou da revisão geral do artigo. 


\section{Referências}

1. Silva NLP, Dessen MA. Deficiência Mental e Família: Implicações para o Desenvolvimento da Criança. Psicologia: Teoria e Pesquisa 2001; 17(2):133-141.

2. Kozma C. What is Down syndrome? In: Stray-Gundersen K, editor. Babies with Down syndrome - a new parent guide. Bethesda: Woodbine House; 1986. p. 1-21.

3. Casarin S. Aspectos Psicológicos na Síndrome de Down. In: Schwartzman JS, organizador. Síndrome de Down. São Paulo: Mackenzie; 2003.

4. Petean EBL, Suguihura ALM. Ter um irmão especial: convivendo com a síndrome de Down. Rev Brasileira de Educação Especial 2005; 11(3):445-460.

5. Chacon MCM. Aspectos relacionais, familiares e sociais da relação pai-filho com deficiência física. Rev Brasileira de Educação Especial 2011; 17(3):441-458.

6. Fiamenghi GA, Messa AA. Pais, filhos e deficiência: estudos sobre as relações familiares. Psicologia: Ciência e Profissão 2007; 27(2):236-245.

7. Mendes EG, Nunes LROP, Ferreira JR. Diagnóstico e caracterização de indivíduos com necessidades educacionais especiais: produção científica nacional entre 1981 e 1998. Temas em Psicologia da SBP 2002; 10(1):11-26.

8. Turnbull AP, Turnbull HR. Families professionals and exceptionality: A special partnership. $2^{\mathrm{a}}$ ed. Columbus: Merrill Publishing Company; 1990.

9. Nunes CC, Silva NVB, Aiello ALR. As Contribuições do Papel do Pai e do Irmão do Indivíduo com Necessidades Especiais na Visão Sistêmica da Família. Psicologia: Teoria e Pesquisa 2008; 24(1):37-44

10. Powell TH, Ogle PA. Irmãos especiais: Técnicas de orientação e apoio para o relacionamento com o deficiente. São Paulo: Editorial Norma; 1992.

11. Silva MTCG. As relações afetivas desenvolvidas entre as pessoas portadoras da síndrome de Down e seus irmãos [dissertação]. São Paulo: Universidade Presbiteriana Mackenzie; 1996.

12. König LG. Irmãos especiais: os portadores de necessidades especiais e suas díades. Monografia [dissertação]. Santa Maria: Universidade de Santa Maria; 1998.

13. Van Riper M. Family variables associated with wellbeing in siblings of children with Down syndrome. Journalof Family Nursing 2000; 6(3):267-286.

14. Nunes CC, Aiello ALR. Interação entre irmãos: deficiência mental, idade e apoio social da família. Psicologia: Reflexão e Crítica 2008; 21(1):42-50.

15. Messa AA, Fiamenghi GA. O impacto da deficiência nos irmãos: histórias de vida. Cien Saude Colet 2010; 15(2):529-538.

16. Frank N. Helping families support siblings. In: Beckman PJ, editor. Strategies for working with families of young children with disabilities. Baltimore: Paul Brookes Publishing; 2000. p. 169-188.

17. Hallahan DP, Kauffman JM. Exceptional Learners: Introduction to Special Education. Yorkshire: Pearson Education; 2000.

18. Hastings RP. Behavioral adjustment of siblings of children with autism engaged in applied behavior analysis early intervention programs: The moderating role of social support. J Autism Dev Disord. 2003; 33(2):141150.

19. Lüdke M, André MEDA. Pesquisa em educação: abordagens qualitativas. São Paulo: EPU; 1986.
20. Manzini EJ. A entrevista na pesquisa social. Didática1990/1991; 26/27:149-158.

21. Vilelas J. Investigação - o Processo de Construção do Conhecimento. Lisboa: Edições Sílabo; 2009.

22. Buscaglia L. O Papel da Família. In: Buscaglia L, organizador. Os deficientes e seus Pais - um desafio ao aconselhamento. Rio de Janeiro: Record; 2006. p. 77-90.

23. Nixon CL, Cummings EM. Siblings disability and children's reactivity to conflicts involving family members. Am Psychol Assoc 1999; 13(2):274-285.

24. Cuskelly M, Gunn P. Sibling relationships of children with Down syndrome: Perspectives of mothers, fathers and siblings. Am J Ment Retard 2003; 108(4):234-244.

25. Núñez B. La família com um hijo com discapacidad: sus conflictos vinculares. Arch Argent Pediatr 2003; 101(2):133-142.

26. Losso R. Psicoanálisis de la familia: recorridos teóricosclínicos. Buenos Aires: Lumen; 2001.

27. Mayer MGG. Síndrome de Down versus alteração de linguagem: interação comunicativa entre pais e filhos [dissertação]. São Carlos: Universidade Federal de São Carlos; 2010.

28. Regen M. Mães e filhos especiais: relato de experiência com grupos de mães de crianças com deficiência. Brasília: CORDE; 1993.

29. Biff LD, Contreras PS, Duarte M. Jovens e Adultos com Síndrome de Down: O Direito à Participação Social. Anais do $22^{\circ}$ Congresso de Iniciação Cientifica UFSCar, 2014.

30. Contreras PS, Duarte M. Experiência de um grupo de orientação e informação para familiares de pessoas com síndrome de Down. Anais do $22^{\circ}$ Congresso de Iniciação Científica UFSCar, 2014.

31. Cuskelly M. Adjustment of siblings of children with a disability: Methodological issues. International Journal for the Advancement of Couselling. V. 21, 111-124, 1999.

32. Lobato D. Brothers, sisters, and special needs: Information and activities for helping young siblings of children with chronic illnesses and developmental disabilities. Baltimore: Paul H. Brookes; 1990.

33. Volling BL, Blandon AY. Positive indicators of sibling relationship quality: Psuchometric analyses of the sibling inventor of behavior. Paper prepared for the Child Trends' Positive Outcomes Conference, March, p. 12-13, 2003.

34. Naylor A, Prescott P. Invisible children? The need for support groups for siblings of disabled children. $\mathrm{Br} \mathrm{J}$ Spec Educ 2004; 31(4):199-206.

35. Brunhara F, Petean EBL. Mães e filhos especiais: reações, sentimentos e explicações à deficiência da criança. Paidéia 1999; 9(16):31-40.

Artigo apresentado em 11/01/2016

Aprovado em 27/06/2016

Versão final apresentada em 29/06/2016 
